\section{Percepção das condições de saúde bucal por gestantes atendidas em uma unidade de saúde no município de Araraquara, São Paulo, Brasil}

\section{Oral health perception of pregnant women seen at a healthcare center in the municipality of Araraquara, São Paulo, Brazil}

Silvio Rocha Corrêa da Silva 1 Fernanda Lopez Rosell 2 Aylton Valsecki Júnior 3

${ }^{1}$ Departamento de Odontologia Social. Faculdade de Odontologia de Araraquara. Universidade Estadual Paulista. Rua Humaitá, 1680, Araraquara, SP, Brasil. CEP: 14.801-903

E-mail: silvio@foar.unesp.br

2-3 Departamento de Odontologia Social. Faculdade de Odontologia de Araraquara. Universidade Estadual Paulista.

\begin{abstract}
Objectives: to assess oral healthcare conditions of a group of pregnant women through the application of the General Oral Health Assessment Index (GOHAI).

Methods: fifty three pregnant women clients of the Basic Oral Health Clinic in Araraquara São Paulo were enrolled in the program. A questionnaire with the GOHAI rating questions, questions related to selfperception of oral conditions and to social and demographic characteristics was applied. Through these Mann-Whitney and Kruskall-Wallis non-paramedic tests, the association of social variables and selfperception of the GOHAI rates were determined.

Results: oral conditions perception as measured by the GOHAI index was positive and presented a median value of 31,6. Subjective data indicated that only 12,0\% of the pregnant women rated their oral condition as "bad", the majority did not disclose any dental problem, although 58,7\% reported gum problems. Issues such as pain and/or discomfort were the ones more closely perceived by the pregnant women.

Conclusions: pregnant women had a positive assessment of their oral conditions with the GOHAI rating associated to self-perception variables. This index could be applied to population groups such as pregnant women, enabling educational and/or preventive measures focused on their real needs.
\end{abstract}

Key words Self-perception, Oral health, Pregnant women

\section{Resumo}

Objetivos: avaliar a percepção das condições de saúde bucal de um grupo de gestantes, através da aplicação do índice General Oral Health Assessment Index (GOHAI).

Métodos: participaram do estudo 53 gestantes que freqüentavam uma Unidade Básica de Saúde em Araraquara, São Paulo. Foi aplicado um questionário contendo questões do índice GOHAI, questões sobre a autopercepção das condições bucais e sobre as características sócio-demográficas. Por meio dos testes nãoparamétricos Mann-Whitney e Kruskall-Wallis, foram determinados a associação das variáveis sociais e de autopercepção com o índice GOHAI.

Resultados: a percepção das condições bucais, medida pelo índice GOHAI, foi positiva e apresentou um valor médio de 31,6. Os dados subjetivos mostram que apenas 12,0\% das gestantes classificaram sua condição bucal como "ruim", a maioria declarou nenhum problema dentário, embora 58,7\% tenha relatado distúrbios gengivais. As questões como dor elou desconforto foram as mais percebidas pelas gestantes.

Conclusões: as gestantes fizeram uma avaliação positiva de sua condição bucal, estando o índice GOHAI associado a variáveis relacionadas à autopercepção. Tal índice pode ser aplicado em grupos populacionais como as gestantes, possibilitando medidas educativas elou preventivas direcionadas às suas reais necessidades.

Palavras-chave Auto-imagem, Saúde bucal, Mulheres grávidas 


\section{Introdução}

As definições mais atuais sobre saúde rejeitam a noção de que saúde é apenas a ausência de doenças físicas, pois é preciso considerar o contexto físico, o psicológico e o bem-estar social do indivíduo. No entanto, a saúde bucal continua sendo medida através de um modelo baseado nas doenças, com indicadores objetivos e quantitativos. Por isso, a prevalência das doenças bucais continua sendo descrita em várias populações, mas pouco se sabe sobre como as doenças e os sintomas afetam o dia-adia das pessoas e sua qualidade de vida. ${ }^{1}$

A importância de se entender como a pessoa percebe sua condição bucal está no fato de que seu comportamento é condicionado por essa percepção, pela importância dada a ela, pelos seus valores culturais e experiências passadas no sistema de saúde. Mesmo nos países mais desenvolvidos e que oferecem serviços odontológicos a sua população, uma grande parcela não os freqüenta porque não tem percepção de sua necessidade. ${ }^{2}$

Para a coleta de dados subjetivos, alguns autores ${ }^{3-5}$ desenvolveram e testaram indicadores que avaliam problemas funcionais, sociais e psicológicos decorrentes das doenças bucais. Embora esses índices apresentem a mesma base conceitual e seus conteúdos sejam similares, eles diferem em tamanho, alcance e complexidade. Assim, enquanto alguns indicadores são compostos por vários itens e subitens, em outros se obtém apenas um valor único.

Este estudo tem por objetivo avaliar a autopercepção das condições de saúde bucal em gestantes que freqüentam uma Unidade Básica de Saúde no município de Araraquara, São Paulo.

Foi escolhido o grupo de gestantes, pois a gestação é um período especial na vida da mulher, onde os cuidados com a saúde devem ser maiores e no qual ela torna-se mais sensível à adoção de novos hábitos e comportamentos. Por isso mesmo, talvez seja o momento mais adequado para analisar como ela percebe sua condição bucal, e a partir daí desenvolver programas educativos e/ou preventivos direcionados às suas reais necessidades.

Costa et al.$^{6}$ reforçam:

"[...] a necessidade da implantação de programas preventivos-educativos, de maneira que a mãe/gestante possa atuar como agente multiplicador de informações que visem à promoção da sua saúde e toda a sua família".

Para Costa et al. 6 estes programas são importantes para que a mãe/gestante passe a ser um agente multiplicador de informações sobre saúde e desmistifique a atenção odontológica.7 No entanto, Romero e Sanches, 8 observaram uma falta de moti- vação da gestante para com os aspectos preventivos odontológicos, resultantes da baixa intervenção educacional por parte do cirurgião-dentista.

A avaliação da percepção das condições bucais da gestante é de fundamental importância para o planejamento e execução de serviços odontológicos voltados para a prevenção e controle desse grupo populacional, tornando possível o desenvolvimento de programas educativos específicos.

\section{Métodos}

Este estudo foi caracterizado como do tipo transversal descritivo, e teve seu início após ser aprovado pelo Comitê de Ética em Pesquisa da Faculdade de Odontologia de Araraquara - Universidade Estadual de São Paulo (UNESP), São Paulo, Brasil, cujo Processo 17/01 foi aprovado em 19 de março de 2004.

A população de estudo foi composta por 53 gestantes que frequentaram, no ano de 2002, uma Unidade Básica de Saúde em Araraquara, para a realização de pré-natal, e consentiram em participar. Trata-se de uma amostra de conveniência devido à facildade de acesso aos sujeitos da pesquisa.

A pesquisa foi realizada através da aplicação de questionário contendo 22 questões. As variáveis de estudo incluídas em um formulário tiveram como objetivo detectar a percepção do indivíduo sobre sua saúde bucal e, por isso, das 22 questões do questionário, 12 eram referentes ao índice General Oral Health Assessment Index (GOHAI) e as outras 10 referentes à auto-avaliação e as características sóciodemográficas da população.

O índice GOHAI, desenvolvido nos Estados Unidos e originalmente desenhado para aplicação em idosos, ${ }^{3}$ vem sendo amplamente utilizado ${ }^{1,9,10} \mathrm{e}$ seus autores, em 1997, defenderam o seu uso também em adultos. Esse índice foi traduzido para o idioma português e validado em população de idosos no Brasil. ${ }^{11}$ Também já foi testado em população de adultos trabalhadores. 12

As questões do índice GOHAI envolvem a análise de informações proporcionadas pelos próprios indivíduos quanto à influência de seus problemas de saúde bucal nas dimensões: a) física/funcional: incluindo alimentação, fala e deglutição (questões 8-12); b) psicossocial/psicológica, compreendendo preocupação ou cuidado com a própria saúde bucal, insatisfação com a aparência, autoconsciência relativa à saúde bucal e o fato de evitar contatos sociais devido a problemas odontológicos (questões 2,3,4,6 e 7) e c) dor/desconforto: 
considerando o uso de medicamentos para aliviar essas sensações, desde que provenientes da boca (questões 1 e 5).13

Para as respostas dessas questões existem pesos/escores que somados darão o valor do índice, o qual quanto maior, melhor a percepção da condição bucal. O índice tem valor máximo de $36 \mathrm{e}$ mínimo de 12 .

As perguntas referentes a auto-avaliação foram formuladas com o objetivo de se obter da gestante as seguintes informações: se ela apresentava, no momento, algum problema nos dentes, na gengiva e qual a nota que ela daria para a sua condição bucal. Essas informações se constituem em um importante indicador de saúde, pois sintetizam desde a condição de saúde objetiva até respostas subjetivas, valores e expectativas clturais.

Também foi definida a condição de classe social das gestantes a partir das questões referentes às condições socioeconômicas, como profissão, ocupação profissional e escolaridade da entrevistada e da pessoa responsável pelo sustento da família. A análise dessas questões e a construção das classes sociais foram realizadas tendo como referência o esquema proposto por Bronfman e Tuirán, adaptado por Lombardi et al.,14 que classifica as pessoas como pertencentes a: burguesia (constituída por proprietários de meios de produção que, sem estar eles mesmos sujeitos à exploração, empregam força de trabalho assalariada); nova pequena burguesia (engloba os agentes sociais que ocupam os postos de mais alto nível técnico e de tomada de decisões); pequena burguesia tradicional (composta pelos agentes sociais que, sem possuir formação univer- sitária, possuem a capacidade de reproduzir-se de maneira independente, por disporem de meios de produção próprios); proletariado típico (composto por trabalhadores que desempenham atividades diretamente vinculadas com a produção e o transporte de mercadorias); proletariado não-típico (formado por assalariados que somente têm relação indireta com a produção) e subproletariado (inclui todos os agentes sociais que desempenham uma atividade, predominantemente não assalariada, em geral instável).

A apuração dos resultados do questionário foi realizada através do software estatístico Epi-info, versão 6.04.15 Em uma primeira etapa, realizou-se a distribuição de freqüência de todas as variáveis de estudo para a confecção de tabelas.

Os testes estatísticos realizados tiveram como objetivo determinar a associação entre o GOHAI e as variáveis sócio-demográficas e subjetivas. Para determinar a significância entre os grupos foram utilizados os testes não-paramétricos Mann-Whitney, quando havia duas amostras independentes, e Kruskal-Wallis, no caso de três ou mais amostras independentes.

A consistência interna e a homogeneidade entre as perguntas do GOHAI foram avaliadas através do cálculo do teste Cronbach's $\alpha$.

\section{Resultados}

Os resultados referem-se aos 53 indivíduos que consentiram participar do estudo. A idade média das gestantes foi de 24,1 anos ( $\mathrm{dp}=6,1$ anos), sendo que a menor idade encontrada foi de 15 anos e a maior

Tabela 1

Questões do índice Geriatric Oral Health Assessment Index (GOHAI) e distribuição de freqüência das respostas em porcentagem. Araraquara, São Paulo, 2002.

\begin{tabular}{|c|c|c|c|c|c|c|c|}
\hline \multirow[t]{2}{*}{ Nos últimos três meses } & \multicolumn{2}{|c|}{$\begin{array}{l}\text { Sempre - } \\
\text { Freqüentemente }\end{array}$} & \multicolumn{2}{|c|}{$\begin{array}{l}\text { Algumas } \\
\text { vezes }\end{array}$} & \multicolumn{2}{|c|}{$\begin{array}{l}\text { Nunca - } \\
\text { Raramente }\end{array}$} & \multirow{2}{*}{$\begin{array}{c}\text { Tota } \\
\mathrm{N}\end{array}$} \\
\hline & $\mathrm{n}$ & $\%$ & $\mathrm{n}$ & $\%$ & $\mathrm{n}$ & $\%$ & \\
\hline 1. Você sentiu seus dentes ou a gengiva ficarem sensíveis a alimentos ou líquidos? & 2 & 3,8 & 17 & 32,1 & 34 & 65,1 & 53 \\
\hline 2. Você evitou comer junto com outras pessoas por causa dos problemas na sua boca? & 1 & 1,9 & 25 & 47,2 & 27 & 50,9 & 53 \\
\hline 3. Você chegou a se sentir nervoso por causa dos problemas na sua boca? & - & - & 9 & 17,0 & 44 & 83,0 & 53 \\
\hline 4. Você teve algum problema na boca que o deixou preocupado? & - & - & 3 & 5,7 & 50 & 94,3 & 53 \\
\hline 5. Você teve que tomar remédio para passar a dor ou o desconforto de sua boca? & 1 & 1,9 & 22 & 41,5 & 30 & 56,6 & 53 \\
\hline 6. Você se sentiu satisfeito ou feliz com a aparência de sua boca? & 49 & 92,4 & 4 & 7,6 & - & - & 53 \\
\hline 7. Você deixou de se encontrar com outras pessoas por causa de sua boca? & 16 & 32,0 & 17 & 34,0 & 17 & 34,0 & 50 \\
\hline 8. Você sentiu algum desconforto ao comer algum alimento? & 4 & 7,7 & 13 & 25,0 & 35 & 37,3 & 52 \\
\hline 9. Você mudou o jeito de falar por causa dos problemas de sua boca? & 3 & 5,7 & 20 & 37,7 & 30 & 56,6 & 53 \\
\hline 10. Você teve dor ou desconforto para engolir os alimentos? & - & - & - & - & 52 & 100,0 & 52 \\
\hline 11. Você teve problemas para mastigar os alimentos? & - & - & 6 & 11,5 & 46 & 88,5 & 52 \\
\hline $\begin{array}{l}\text { 12. Você diminuiu a quantidade de alimentos ou mudou o tipo de alimentação } \\
\text { por causa de seus dentes? }\end{array}$ & 6 & 11,3 & 25 & 45,3 & 22 & 43,4 & 53 \\
\hline
\end{tabular}


Valores médio, mínimo e máximo do índice GOHAl segundo as variáveis demográficas e de autopercepção na amostra de gestantes. Araraquara, São Paulo, 2002.

\begin{tabular}{|c|c|c|c|c|c|}
\hline \multirow[t]{2}{*}{ Características } & \multirow[t]{2}{*}{$\mathbf{N}$} & \multirow[t]{2}{*}{$\%$} & \multicolumn{3}{|c|}{ GOHAI } \\
\hline & & & Média & Mínimo & Máximo \\
\hline Gestantes & 53 & 100 & 31,6 & 22 & 36 \\
\hline \multicolumn{6}{|l|}{ Idade } \\
\hline 15 I-- 20 & 10 & 19,2 & 31,0 & 25 & 36 \\
\hline $201--25$ & 20 & 38,5 & 32,5 & 27 & 36 \\
\hline $251--30$ & 16 & 28,8 & 31,3 & 22 & 36 \\
\hline 30 I-- 35 & 5 & 9,6 & 30,0 & 25 & 35 \\
\hline 35 e + & 2 & 3,9 & 28,0 & 26 & 30 \\
\hline \multicolumn{6}{|l|}{ Anos de estudo* } \\
\hline$\leq 8$ & 27 & 50,9 & 30,9 & 22 & 36 \\
\hline$>8$ & 26 & 49,1 & 31,9 & 25 & 36 \\
\hline \multicolumn{6}{|l|}{ Classe social** } \\
\hline Subproletariado & 2 & 4,5 & 32,0 & 32 & 32 \\
\hline Proletariado típico & 29 & 65,9 & 31,1 & 24 & 36 \\
\hline Proletariado não-típico & 9 & 20,5 & 31,9 & 22 & 36 \\
\hline Pequena burguesia tradicional & 3 & 6,8 & 31,0 & 28 & 35 \\
\hline Nova pequena burguesia & 1 & 2,3 & 35,0 & 35 & 35 \\
\hline \multicolumn{6}{|l|}{ Auto-avaliação da condição bucal** } \\
\hline Excelente & 5 & 10,0 & 35,4 & 35 & 36 \\
\hline Boa & 16 & 32,0 & 31,3 & 25 & 36 \\
\hline Regular & 23 & 46,0 & 30,6 & 28 & 35 \\
\hline Ruim & 6 & 12,0 & 26,8 & 22 & 31 \\
\hline Péssima & 0 & 0,0 & - & - & - \\
\hline \multicolumn{6}{|l|}{ Problemas com os dentes* } \\
\hline $\operatorname{sim}$ & 14 & 28,0 & 31,9 & 25 & 35 \\
\hline Não & 36 & 72,0 & 31,2 & 22 & 36 \\
\hline \multicolumn{6}{|l|}{ Problemas com a gengiva* } \\
\hline Sim & 27 & 58,7 & 31,2 & 25 & 36 \\
\hline Não & 19 & 41,3 & 29,7 & 22 & 35 \\
\hline
\end{tabular}

* $p<0,001$ (teste Mann-Whitney) ** $p<0,001$ (teste Kruskall-Wallis)

de 51 anos, com mediana de 23 anos.

Em média as gestantes estudaram 8,6 anos e com relação a classe social, $65,9 \%$ foram classificadas como pertencentes ao proletariado típico; $20,5 \%$ ao proletariado não-típico; $6,8 \%$ a pequena burguesia tradicional; $4,5 \%$ ao subproletariado e $2,3 \%$ a nova pequena burguesia, segundo a classificação de classe social adotada neste estudo.

Sobre a percepção das suas condições bucais, $72,0 \%$ das gestantes declararam não ter problemas com os dentes, mas em compensação, 58,7\% delas acreditavam que possuíam algum problema na gengiva. Quarenta e seis por cento das gestantes achavam que sua condição bucal era regular, para $32 \%$ a condição era boa, para $12 \%$ era ruim e para $10 \%$ excelente.

As questões do índice GOHAI e a distribuição de respostas para a amostra estudada estão na Tabela 1 , onde podem ser observadas as três dimensões do índice: problemas físicos e/ou funcionais, problemas psicossociais e dor e/ou desconforto.

O teste Cronbach's $\alpha(0,82)$ mostrou alto grau de consistência interna e de homogeneidade entre as perguntas.

Na Tabela 2 são mostrados os valores médio, mínimo e máximo do índice GOHAI, de acordo com 
as características demográficas e de autopercepção das gestantes, e se há diferença estatisticamente significante ente os valores das variáveis e o índice GOHAI.

\section{Discussão}

A caracterização social da amostra estudada mostrou uma população jovem, média de 24,1 anos de idade, com 8,6 anos de estudo, em média, o que evidência que, pelo menos, o ensino fundamental foi completado. As classes sociais predominantes foram o proletariado típico e o proletariado não-típico, que corresponde a uma situação onde o indivíduo, sem possuir curso superior, vende sua força de trabalho em troca de um salário.

Considerou-se importante classificar esses indivíduos segundo sua classe social, pois as doenças que afetam os indivíduos não podem ser explicadas somente pelos fatores biológicos que as caracterizam, uma vez que a qualidade de vida, decorrente dos aspectos sociais, econômicos, políticos e culturais de uma sociedade, é determinante essencial.

Sabe-se que as condições de vida e de trabalho qualificam de forma diferenciada a maneira pela qual os indivíduos pensam, sentem e agem a respeito de sua saúde. Segundo Bastos et al.16:

"[...] classificar a saúde em boa, má ou razoável é também definir a qualidade de vida, pois ela surge das condições de classe social, das relações no trabalho, da alimentação, da moradia, do saneamento básico, do meio ambiente saudável, do acesso à educação, ao transporte, ao lazer, aos serviços de saúde, enfim, tudo o que diz respeito à vida"

Como grande parte das entrevistadas se declararam donas de casa e viviam com alguém que as sustentava, as informações sobre esses indivíduos foram importantes na determinação das classes sociais. O marido foi referido como o chefe da família por $71,1 \%$ das gestantes e $72,5 \%$ destas estavam sem emprego no momento da pesquisa.

Sobre a percepção da condição bucal as gestantes fizeram uma avaliação positiva, pois $42 \%$ delas classificaram-na como "boa" ou "excelente" e $46 \%$ como "regular", aproximando-se dos valores encontrados por Silva12 em população adulta de trabalhadores. Em relação ao GOHAI, houve associação positiva com indivíduos que avaliaram sua condição bucal como regular ou boa, dados similares aos de outros estudos. $3,11,12$

Declararam não ter nenhum problema com seus dentes $72,0 \%$ das entrevistadas, enquanto as doenças que ocorrem na gengiva foram relatadas por $58,7 \%$ delas. Esses valores são maiores do que outros encontrados na literatura, principalmente em relação à percepção dos problemas na gengiva, pois vários estudos relatam baixa percepção dos problemas periodontais. Silva e Fernandes 11 encontraram, em idosos, apenas $18,8 \%$ de percepção desses problemas, enquanto Silva12 relatou, em adultos, $31,0 \%$ de percepção dos problemas gengivais. Houve associação significativa entre o GOHAI e a percepção desses dois problemas bucais.

O índice GOHAI foi desenvolvido e testado nos Estados Unidos ${ }^{3}$ em população com características sociais, econômicas e culturais bem diferentes das gestantes que procuraram o Centro de Saúde em Araraquara. $\mathrm{O}$ índice tem um escore que varia de 12 a 36 e quanto maior o valor melhor a condição bucal, sendo encontrado entre as gestantes um valor médio de 31,6. Outros autores que empregaram esse índice, no Brasil, encontraram valores próximos ao referido acima. Assim, Silva,12 estudando adultos trabalhadores em Diadema, São Paulo, obteve um GOHAI de 32,5, enquanto Silva e Fernandes, 11 em idosos de Araraquara, São Paulo, encontraram um índice de 33,8 e Silva, 17 em idosos de Piracicaba, São Paulo, obteve um valor de 28,5.

Embora no presente estudo não tenha sido feito o exame clínico das gestantes e, portanto, não seja possível comparar esses dados subjetivos com a situação clínica das mulheres, estudos sobre autopercepção18 já mostraram que a maioria das pessoas vêem sua condição bucal de maneira favorável mesmo com condições clínicas não satisfatórias, provavelmente porque as medidas clínicas de saúde, utilizadas pelo profissional, são preditores relativamente fracos da percepção de saúde bucal das pessoas.

Alguns autores 2,3 mostraram que existe relação entre a percepção da condição bucal e algumas variáveis clínicas, mas que se trata de uma associação relativamente fraca. De acordo com Jokovic e Locker, 18 uma das razões para a fraca associação entre as variáveis clínicas e a auto-avaliação deve-se ao fato de que muitas das doenças detectadas no exame clínico são assintomáticas e provavelmente desconhecidas do indivíduo. Para Locker e Miller,5 saúde é um estado percebido subjetivamente e a validade da autopercepção não deve estar baseada na sua associação ou ausência de associação com os indicadores clínicos. Atchison e Dolan ${ }^{3}$ afirmaram que a percepção é uma medida muito mais de "saúde" do que de "morbidade", que é a base das avaliações clínicas, e, portanto, é necessário reconhecer que medidas de saúde e medidas de doenças são qualitativamente diferentes e têm implicações diferentes. 
Por isso, indicadores subjetivos, como o GOHAI, podem ser importantes por estarem, geralmente, correlacionados com a autopercepção e serem indicadores de necessidades de atenção. Devem, sempre que possível, ser incluídos em estudos que avaliem condições de saúde bucal, pois embora não cumpram o papel de identificar todos os indivíduos que tenham necessidades de tratamento, podem ser úteis na identificação de estratos populacionais que necessitam de prioridade na atenção.

Considerando-se que a mãe tem um importante papel na família, especialmente se a questão é sobre saúde, e que a gestação se constitui em um período no qual ela se encontra receptiva à incorporação de novas atitudes e comportamentos, conclui-se ser extremamente importante conhecer como ela percebe sua condição bucal, pois é a partir dessa percepção que o indivíduo molda o seu comportamento. Para os serviços de saúde é a partir desse

\section{Referências}

1. Tubert-Jeannin S, Riordan PJ, Morel-Papernot A, SabyCollet S. Validation of an oral health quality of life index (GOHAI) in France. Community Dent Oral Epidemiol 2003; 31: 275-84.

2. Steele JG, Walls AWG, Ayatollahi SMT, Murray JJ. Major clinical findings from a dental survey of elderly people in three different English communities. Br Dent J 1996; 180: 17-23.

3. Atchison KA, Dolan TA. Development of the Geriatric Oral Health Assessment Index. J Dental Educ 1990; 54: 680-7.

4. Leão A, Sheiham A. Relation between clinical dental status and subjective impacts on daily living. J Dent Res 1995; 74: 1408-13.

5. Locker D, Miller Y. Evaluation of subjective oral health status indicators. J Public Health Dent 1994; 54: 167-76.

6. Costa ICC, Marcelino G, Berti Guimarães M, Saliba NA. A gestante como agente multiplicador de saúde. RPG Rev Pos-Grad 1998; 5: 87-92.

7. Costa ICC, Saliba O, Moreira ASP. Atenção odontológica à gestante na concepção médico-dentista-paciente: representações sociais dessa interação. RPG Rev Pos-Grad 2002; 9: 232-43.

8. Romero FD, Sanches CM. Los odontologos educan a sus pacientes? Rev ADM 1988; 45: 317-20.

9. Locker D, Matear D, Stephens M, Jokovic A. Oral healthrelated quality of life of a population of medically compromised elderly people. Community Dent Health 2002; 19 : 90-7.

10. Wong MC, Liu JK, Lo EC. Translation and validation of the Chinese version of GOHAI. Public Health Dent 2002; 62: 78-83. conhecimento que podem ser desenvolvidos programas educativos visando maior esclarecimento das pessoas.

\section{Considerações finais}

De acordo com os resultados obtidos e análises realizadas neste estudo, concluiu-se que as gestantes que freqüentaram uma Unidade Básica de Saúde em Araraquara fizeram uma avaliação positiva de sua condição bucal, com o índice GOHAI estando associado as variáveis relacionadas à autopercepção. Esses resultados mostram que o índice pode ser aplicado em outros grupos populacionais, além de idosos, e que pode ser importante no desenvolvimento de programas educativos e preventivos específicos a determinados grupos populacionais.

11. Silva SRC, Fernandes RAC. Autopercepção das condições de saúde bucal por idosos. Rev Saúde Pública 2001; 35 : 349-55.

12. Silva DRAD. Percepção das condições de saúde bucal em adultos trabalhadores. [dissertação mestrado]. São Paulo: Faculdade de Saúde Pública da Universidade de São Paulo; 2000.

13. Kressin NR, Atchison KA, Miller DR. Comparing the impact of oral disease in two populations of older adults: application of the geriatric oral health assessment index. J Public Health Dent 1997; 57: 224-32.

14. Lombardi C, Bronfman M, Facchini LA, Victora CG, Barros FC, Beria JU. Operacionalização do conceito de classe social em estudos epidemiológicos. Rev Saúde Pública 1988; 22: 253-65.

15. Dean AG, Dean JA, Coulombier D, Brendel KA, Smith DC, Burton $\mathrm{AH}$, et al. Epi Info, version 6:a word processing, database, and statistics program for epidemiology on microcomputers. Atlanta, Georgia: Centers for Disease Control and Prevention; 1994.

16. Bastos JRM, Saliba NA, Unfer B. Considerações a respeito de saúde bucal e classes sociais. Rev Paul Odontol 1996; 18: $38-42$.

17. Silva DD. Aspectos epidemiológicos e autopercepção da saúde bucal em idosos. [dissertação mestrado]. Piracicaba: Faculdade Odontologia de Piracicaba da Universidade Estadual de Campinas; 2003.

18. Jokovic A, Locker D. Dissatisfaction with oral health status in an older adult population. J Public Health Dent 1997; 57 : 40-7.

Recebido em 7 de Abril de 2005

Versão final apresentada em 07 de Novembro de 2006

Aprovado em 16 de novembro de 2006 